\title{
PREHEATING AND PHASE TRANSITIONS IN GAUGE THEORIES *
}

\author{
A. RAJANTIE \\ Centre for Theoretical Physics, University of Sussex, Brighton BN1 9QH, UK ${ }^{\dagger}$
}

\begin{abstract}
It has recently been suggested that the baryon washout problem of the standard electroweak baryogenesis scenario could be avoided if inflation ends with a period of parametric resonance at a low enough energy density. I present results of numerical simulations in which this process was studied in the Abelian Higgs model. Our results show that because of the masslessness of the gauge field, the parametric resonance takes place naturally, and that the system reaches a quasi-equilibrium state in which the long-wavelength part of the spectrum has a high effective temperature. This enhances baryon number violation and makes baryogenesis more efficient.
\end{abstract}

\section{Introduction}

If $\mathrm{CP}$ is violated at high energies by some physics beyond the standard model, the electroweak theory and the standard Big Bang scenario seem to contain all the ingredients for explaining the baryon asymmetry of the universe 1 The necessary out-of-equilibrium conditions are provided by the dynamics of the phase transition and the last one of the three Sakharov conditions, number violation, is satisfied by sphaleron processes, which change the ChernSimons number and consequently, due to a quantum anomaly, also the baryon number.

However, although sphaleron processes become less frequent after the phase transition, they don't disappear completely. Instead, their rate is proportional to $\exp \left(-M_{\mathrm{sph}} / T\right)$, where $M_{\mathrm{sph}} \propto \phi$ and $\phi$ is the expectation value of the Higgs field, and unless $\phi$ is large enough, the baryon asymmetry generated in the transition can be washed out. This can only be avoided if the transition is strongly enough first order so that the discontinuity of the Higgs field is $\Delta \phi \gtrsim T$.

In the minimal standard model, the Higgs mass $m_{H}$ is the only unknown parameter, and lattice simulation 3 have revealed that, whatever its value, the transition is not strong enough. In more complicated models, such as MSSM, there are more unknown parameters and this freedom makes it possible to satisfy the constraint, but only barely.

\footnotetext{
*Talk presented at SEWM2000, Marseille, France, 14-17 June, 2000.

${ }^{\dagger}$ Present address: DAMTP, CMS, Wilberforce Road, Cambridge CB3 0WA, UK
} 
In an alternative scenario proposed recently by two groups, 明 the electroweak baryogenesis takes place during a period of preheating after inflation. This requires that inflation ends at an energy scale that is below the electroweak scale and that a large fraction of the energy of the inflaton is transferred rapidly to the standard model fields by a parametric resonance 1 In the resulting non-equilibrium power spectrum, all the fermionic fields and the short-wavelength modes of the bosons are practically in vacuum, but the long-wavelength bosonic modes have a high energy density. The sphaleron rate depends strongly on the temperature of these long-wavelength modes and is therefore very high, and the out-of-equilibrium processes can generate a large baryon asymmetry very quickly. Eventually the system equilibrates and the effective temperature decreases by a rate determined by the decay rate of gauge bosons into fermions, $\Gamma \sim 1 \mathrm{GeV}$. The final temperature $T_{\text {reheat }}$ is determined by the initial energy density and provided that it is low enough, $T_{\text {reheat }} \lesssim 0.5 T_{c}$, the sphaleron rate becomes negligible and the baryon washout is avoided.

In this talk, I will discuss the recent numerical simulations 1 in which this process was studied in the Abelian Higgs model.

\section{Simulations}

Instead of considering any particular model of inflation, we simply assume that the inflaton interacts mostly with the Higgs, and that from the point of view of the gauge fields, we can describe the Higgs and the inflaton by a single scalar degree of freedom, which has a large energy density in its longwavelength modes, which is realized by giving the scalar field $\phi$ a large initial value $\phi_{0}$. Since very little is known about the details of the inflaton, it is difficult to improve this approximation.

When inflation ends, the inflaton field is typically still quite far away from its minimum and contains a large amount of energy. Because inflation dilutes all inhomogeneities, this energy is concentrated in the very long-wavelength modes. Moreover, all the standard model fields are in vacuum.

Instead of the full standard model gauge group, we used only a single Abelian gauge field, because that makes the simulation much simpler and is unlikely to change the qualitative behaviour. The Lagrangian of our model is

$$
L=-\frac{1}{4} F^{\mu \nu} F_{\mu \nu}+\left(D^{\mu} \phi\right)^{*} D_{\mu} \phi-\lambda\left(|\phi|^{2}-v^{2}\right)^{2},
$$

where the gauge covariant derivative is $D_{\mu} \phi=\partial_{\mu} \phi+i e A_{\mu} \phi$, and $F_{\mu \nu}=$ $A_{\nu ; \mu}-A_{\mu ; \nu}$. 
Since the occupation numbers of the long-wavelength modes will be high, the dynamics of the system can be approximated by the classical equations of motion. However, the quantum vacuum fluctuations are important as seeds for the parametric resonance, and therefore we approximate them by adding Gaussian fluctuations with the same two-point function as in the quantum vacuum, i.e.

$$
\left\langle\phi^{*}(\vec{k}) \phi\left(\vec{k}^{\prime}\right)\right\rangle=\frac{1}{2 \omega(\vec{k})}(2 \pi)^{3} \delta^{(3)}\left(\vec{k}-\vec{k}^{\prime}\right) .
$$

In a sense, this means that the quantum effects are approximated to leading order in perturbation theory.

In the full electroweak case, the effective temperature of the longwavelength modes decreases mostly because the Higgs and gauge bosons decay into fermions. We approximate this by letting the universe expand according to $a=\sqrt{1+2 H t}$ at the rate $H=\dot{a} / a \approx 0.7 \mathrm{GeV} \sim \Gamma$. This has the effect of reducing the energy in the long-wavelength modes, and if we use the conformal time coordinate $\eta$ defined by $d \eta \equiv d t / a$, it appears simply as a changing mass term for the Higgs field

$$
m^{2}(t)=-2 \lambda v^{2} a^{2}+\partial_{\eta}^{2} a / a .
$$

In the simulation, we used a $240^{3}$ lattice with lattice spacing $\delta x / a=$ $1.4 \mathrm{TeV}^{-1}$ and time step $\delta t / a=0.14 \mathrm{TeV}^{-1}$. The couplings were $e=0.14$, $\lambda=0.04$ and $v=246 \mathrm{GeV}$. The initial value of the Higgs field was $\phi_{0}=1 \mathrm{TeV}$.

\section{Results}

The time evolution of $|\phi|^{2}$ is shown in Fig. 1. (We have subtracted the dominant ultraviolet divergence $\left\langle|\phi|^{2}\right\rangle_{\text {div }} \approx 0.226 / \delta x^{2}$.) This model does not have any local order parameter in the rigorous sense, and $|\phi|^{2}$ in particular is nonzero in both the symmetric and the broken phase. However, Fig. 1 s shows that until $t \approx 7 \mathrm{GeV}^{-1},|\phi|^{2}$ decreases roughly as $a^{-1}$, indicated by the dotted line, which strongly suggests that no Higgs condensate is present and that the system is in the symmetric phase. After that, the condensate develops, and $|\phi|^{2}$ starts to approach its vacuum expectation value.

In the inset of Fig. 1, we have plotted the power spectrum of the electric field in terms of the effective temperature as a function of momentum at various times during the time evolution. The definition of $T_{\text {eff }}$ is

$$
T_{\text {eff }}(k)=\frac{1}{2}\left|E_{i}^{\mathrm{T}}(k)\right|^{2} \frac{d^{3} k}{(2 \pi)^{3}},
$$

proc: submitted to World Scientific on November 5, 2018 


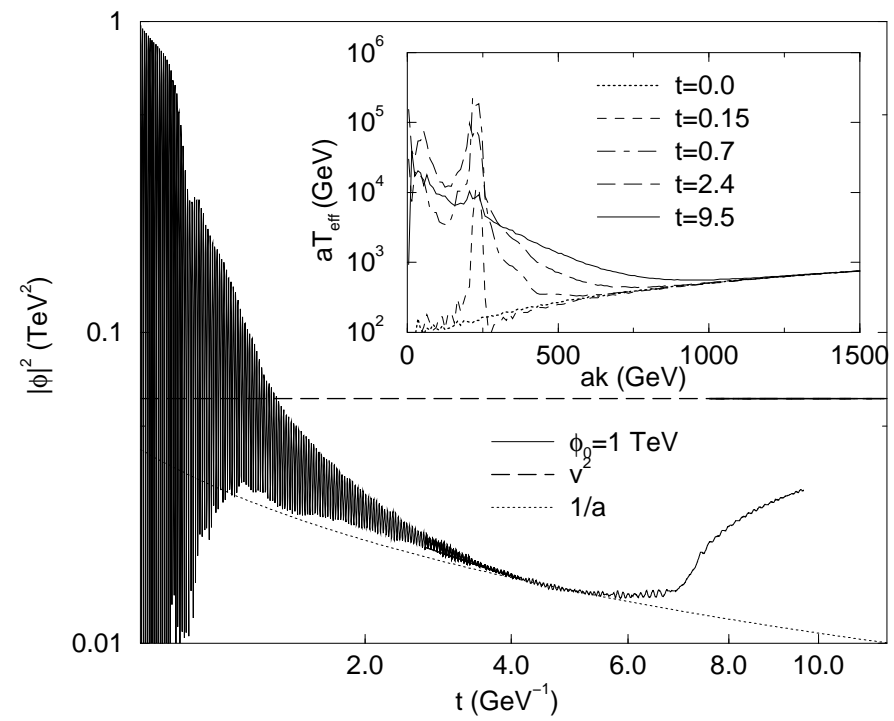

Figure 1. The time evolution of $|\phi|^{2}$ on on a $240^{3}$ lattice with the initial condition $\phi_{0}=1 \mathrm{TeV}$. The symmetry is restored until $t \approx 7 \mathrm{GeV}^{-1}$. The effective temperature of different Fourier modes is shown in the inset. Long-wavelength modes equilibrate at a high temperature, but short wavelengths remain in vacuum.

where the superscript $\mathrm{T}$ indicates the transverse component of the electric field; the longitudinal component is fixed by the Gauss law. In thermal equilibrium at temperature $T, T_{\text {eff }}(k)=T$ for every $k$. We can see that initially, the power spectrum develops a sharp peak, which later spreads, and the power spectrum reaches a quasi-equilibrium form in which the long-wavelength modes $k \lesssim k_{*} \sim e \phi_{0}$ are at a high effective temperature $T_{\text {eff }} \sim \phi_{0} / e$. The short-wavelength modes with $k \gtrsim k_{*}$ remain in vacuum, where the effective temperature due to vacuum quantum fluctuations is $T_{\text {eff }}=k_{*} / 2$. Because of the expansion of the space, the cutoff scale and the effective momentum decrease as $a^{-1}$, but apart from that, the form of the power spectrum remains qualitatively the same until the end of our simulations.

The form of this quasi-equilibrium power spectrum is crucial for the scenario of electroweak baryogenesis at preheating. The sphaleron rate is proportional to $T^{4}$ and is only sensitive to the long-wavelength modes, and as their temperature is high, the baryon number violation is very strong. Furthermore, $\left\langle\phi^{2}\right\rangle$ and $\left\langle A_{i}^{2}\right\rangle$ also have much larger values than they would have 
in equilibrium with the same energy density and since the effective mass of the Higgs field is given by

$$
m_{\phi}^{2} \approx-2 \lambda v^{2}+4 \lambda\left\langle\phi^{2}\right\rangle+e^{2}\left\langle A_{i}^{2}\right\rangle,
$$

the symmetry restoration is much easier. erate the baryon asymmetry during a period of this "non-thermal" symmetry restoration, and since the effective temperature decreases much faster than the baryons can decay, the baryon asymmetry will quickly freeze in.

Our results show that the qualitative behaviour of gauge-Higgs models is compatible with electroweak baryogenesis at preheating. In order to test the scenario quantitatively, we are currently working on simulations in the $\mathrm{SU}(2) \times \mathrm{U}(1)$ theory. 9

\section{Acknowledgments}

I would like to thank E.J. Copeland and P.M. Saffin for collaboration on this subject, and PPARC and the University of Helsinki for financial support. This work was conducted on the SGI Origin platform using COSMOS Consortium facilities, funded by HEFCE, PPARC and SGI.

\section{References}

1. V. A. Kuzmin, V. A. Rubakov and M. E. Shaposhnikov, Phys. Lett. B155 (1985) 36.

2. A. D. Sakharov, JETP Lett. 5 (1967) 24.

3. K. Kajantie, M. Laine, K. Rummukainen and M. Shaposhnikov, Nucl. Phys. B493 (1997) 413 hep-lat/9612006.

4. L. M. Krauss and M. Trodden, Phys. Rev. Lett. 83 (1999) 1502 hep$\mathrm{ph} / 9902420$.

5. J. Garcia-Bellido, D. Y. Grigoriev, A. Kusenko and M. Shaposhnikov, Phys. Rev. D60 (1999) 123504 hep-ph/9902449].

6. J. H. Traschen and R. H. Brandenberger, Phys. Rev. D42, 2491 (1990); L. Kofman, A. Linde, and A. A. Starobinsky, Phys. Rev. Lett. 73, 3195 (1994) hep-th/9405187.

7. A. Rajantie and E. J. Copeland, Phys. Rev. Lett. 85 (2000) 916 hep$\mathrm{ph} / 0003025$.

8. L. Kofman, A. Linde, and A. A. Starobinsky, Phys. Rev. Lett. 76, 1011 (1996) hep-th/9510119]; I. I. Tkachev, Phys. Lett. B376, 35 (1996) hep-th/9510146.

9. A. Rajantie, P.M. Saffin and E.J. Copeland, in progress.

proc: submitted to World Scientific on November 5, 2018 